\title{
Búsqueda de Borrelia spp. en garrapatas del género Amblyomma de Argentina
}

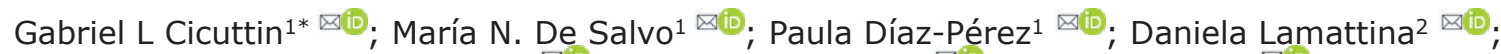

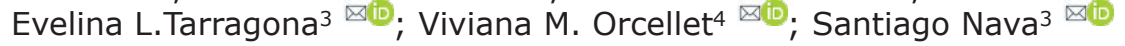

\begin{abstract}
${ }^{1}$ Instituto de Zoonosis Luis Pasteur, Ministerio de Salud del Gobierno de la Ciudad Autónoma de Buenos Aires, Ciudad Autónoma de Buenos Aires, Argentina

2Instituto Nacional de Medicina Tropical, Ministerio de Salud de la Nación, Almafuerte y Ámbar S/N, Misiones, Argentina

${ }^{3}$ Instituto Nacional de Tecnología Agropecuaria, Estación Experimental Agropecuaria Rafaela. Consejo Nacional de Investigaciones Científicas y Técnicas, Rafaela, Santa Fe, Argentina

${ }^{4}$ Universidad Nacional del Litoral, Facultad de Ciencias Veterinarias, Esperanza, Santa Fe, Argentina.

*Correspondencia: gcicuttin@gmail.com
\end{abstract}

Recibido: Octubre 2020; Aceptado: Mayo 2021; Publicado: Junio 2021.

\section{RESUMEN}

Objetivo. El objetivo del presente estudio fue detectar la presencia de Borrelia spp. en especies de garrapatas del género Amblyomma en Argentina. Materiales y métodos. Se analizaron especímenes de garrapatas del género Amblyomma colectadas de distintas provincias de Argentina (Entre Ríos, Misiones, Formosa, Salta, Córdoba, San Luis y Buenos Aires) mediante una PCR anidada para un fragmento de 306 pb del gen fla del género Borrelia. Resultados. Se analizaron 422 ejemplares de las especies Amblyomma aureolatum, Amblyomma brasiliense, Amblyomma ovale, Amblyomma sculptum, Amblyomma tigrinum, Amblyomma tonelliae y Amblyomma triste de diversas regiones de Argentina. Todas las muestras estudiadas resultaron negativas mediante la PCR. Conclusiones. Las especies de garrapatas del género Amblyomma analizadas en el presente estudio son de importancia en salud pública; si bien no se detectó infección con bacterias del género Borrelia, considerando los escasos antecedentes previos, futuros estudios en esta temática son necesarios en este género de garrapatas.

Palabras clave: Borrelia; Amblyomma; Argentina (Fuente: CAB, TGN).

\section{ABSTRACT}

Objetive. The aim of the present study was to detect Borrelia spp. in different species of ticks of the genus Amblyomma from Argentina. Materials and methods. Amblyomma tick specimens collected from various provinces of Argentina (Entre Ríos, Misiones, Formosa, Salta, Córdoba, San Luis and Buenos Aires) were analyzed by nested PCR for a 306 bp fragment of the gene fla of the genus Borrelia. Results. A total of 422 tick specimens (Amblyomma aureolatum, Amblyomma brasiliense, Amblyomma ovale, Amblyomma sculptum, Amblyomma tigrinum, Amblyomma tonelliae and Amblyomma triste) from various regions of Argentina were studied. All samples were negative by PCR for a fragment of the gene fla of Borrelia spp. Conclusions. The tick species of the genus Amblyomma analyzed in the present study are important for public health; although infection with bacteria of the genus Borrelia was not detected. However, considering the few previous antecedents in the region, future studies on this genus of ticks are necessary.

Keywords: Borrelia; Amblyomma; Argentina (Source: CAB, TGN).

Como citar (Vancouver).

Cicuttin GL, De-Salvo MN, Díaz-Pérez P, Lamattina D, Tarragona EL, Orcellet VM, Nava S. Búsqueda de Borrelia spp. en garrapatas del género Amblyomma de Argentina. Rev MVZ Córdoba. 2021; 26(3):e2199. https://doi.org/10.21897/rmvz.2199 CC) 5 ( 9 (los) autor (es), Revista MVZ Córdoba 2021. Este artículo se distribuye bajo los términos de la licencia internacional Creative Commons Attribution BY NC SA comercial, siempre y cuando den crédito y licencien sus nuevas creaciones bajo las mismas condiciones. 


\section{INTRODUCCIÓN}

Las garrapatas del género Amblyomma (Familia Ixodidae) comprenden aproximadamente 135 especies a nivel mundial, siendo el género con mayor riqueza específica en la región neotropical (1). Aunque el rango de hospedadores de las garrapatas del género Amblyomma incluye todas las clases de vertebrados terrestres, la mayoría de las especies presentes en el cono sur de América se alimentan sobre mamíferos, aunque las aves también son importantes hospedadores para los estadios inmaduros de muchas de ellas (1). Diferentes especies de Amblyomma fueron reportadas infestando humanos en el cono sur de América, como por ejemplo, Amblyomma aureolatum (estadios adultos), Amblyomma brasiliense (todos los estadios), Amblyomma coelebs (todos los estadios), Amblyomma dubitatum (todos los estadios), Amblyomma neumanni (todos los estadios), Amblyomma ovale (adultos), Amblyomma parvum (adultos), Amblyomma sculptum (todos los estadios), Amblyomma tigrinum (adultos), Amblyomma tonelliae (todos los estadios) y Amblyomma triste (adultos), todas ellas presentes en Argentina (1). Además, algunas de estas especies actúan como vectores de diversos microorganismos patógenos (p.e. Rickettsia rickettsii y Rickettsia parkeri) para los humanos (1).

El género Borrelia (Familia Spirochaetaceae) comprende más de 30 genoespecies transmitidas por artrópodos con diferente grado de patogenicidad $(2,3)$. Las bacterias del género Borrelia están contenidas en tres grupos: Borrelia burgdorferi sensu lato (GBB), transmitido principalmente por garrapatas del género Ixodes; Fiebre Recurrente (GFR), transmitido por garrapatas y piojos; y un tercer grupo también transmitido por garrapatas (REP) $(3,4)$. El GBB consiste en aproximadamente 18 genoespecies formalmente descriptas, incluyendo los principales agentes de la enfermedad de Lyme: Borrelia burgdorferi sensu stricto, Borrelia afzelii y Borrelia garinii, todas transmitidas por garrapatas del complejo Ixodes ricinus en el hemisferio norte $(2,3)$. Dentro de las borrelias del GFR transmitidas por garrapatas (GFR-TG), existen genoespecies transmitidas por garrapatas blandas (familia Argasidae), p.e., Borrelia hispánica, Borrelia turicatae y Borrelia anserina y genoespecies transmitidas por garrapatas duras (familia Ixodidae), p.e. Borrelia miyamotoi y Borrelia theileri $(2,3)$. Por último, el REP engloba Borrelia spp. transmitidas por garrapatas duras que se han detectado en diferentes regiones del mundo; cabe aclarar que si bien su nombre se debe a que originalmente fue hallado en reptiles o garrapatas asociadas a reptiles, también ha sido detectado en garrapatas duras no relacionadas con reptiles, así como aves $(2,3,4,5)$.

Comparativamente, la asociación de Borrelia spp. con garrapatas del género Amblyomma ha sido menos estudiada respecto a la asociación entre borrelias y garrapatas del género Ixodes. En América, se detectaron genoespecies (Borrelia lonestari) del GFR-TG en Amblyomma americanum de EEUU $(6,7)$ y genoespecies del REP en Amblyomma maculatum de EEUU (4), Amblyomma longirostre de Brasil (5) y Guayana Francesa (8), Amblyomma geayi de Guayana Francesa (8), A. aureolatum de Argentina (3) y Amblyomma dissimile de México (9). Además, recientemente dos Santos y col. reportaron Borrelia sp. de un grupo separado a REP y GFR en $A$. brasiliense (10).

Considerando los escasos estudios sobre el potencial rol de las garrapatas del género Amblyomma como vectores de borrelias en Sudamérica, el objetivo de este trabajo fue analizar la presencia de Borrelia spp. en distintas especies de este género de garrapatas en Argentina.

\section{MATERIALES Y MÉTODOS}

Muestras. Los especímenes de garrapatas utilizados en este estudio, colectados en fase parasitaria y de vida libre, corresponden a material ya publicado en Cicuttin et al. $(11,12,13)$, Lamattina et al (14), Mastropaolo et al (15) y Tarragona et al (16), por lo que los datos de métodos de muestreo, características ambientales de los sitios de colecta y determinación taxonómica se encuentran detallados en los mismos. Las garrapatas colectadas en las provincias de Entre Ríos y Formosa fueron extraídas en forma manual sobre los hospedadores o por método bandera en su fase de vida libre en vegetación, e identificadas siguiendo las descripciones y claves presentadas en Nava et al (1).

Extracción de ADN. Las garrapatas fueron procesadas en forma individual. La extracción de los ácidos nucleicos se realizó mediante AxyPrep Multisource Genomic DNA MiniPrep Kit (Axygen Biosciences, USA), High Pure PCR Template Preparation Kit (Roche, Germany) y mediante fenol-cloroformo-alcohol isoamílico. 
El detalle de la metodología utilizada fue publicada previamente, no se utilizaron controles endógenos $(11,12,13,14,15,16)$.

Detección molecular de Borrelia spp. Se realizó mediante una $\mathrm{PCR}$ anidada para un fragmento de $306 \mathrm{pb}$ del gen fla con los cebadores: Fla-LL 5'-ACATATTCAGATGCAGACAGAGGT-3', Fla-RL 5 '-GCAATCATAGCCATTGCAGATTGT-3', Fla-LS 5' -AACAG-CTGAAGAGCTTGGAATG-3' y Fla-RS $\quad 5^{\prime}$-CTTTGATCACTTTCATTCTAATAGC-3'(6). Se utilizaron como controles $B$. burgdorferi s.s. (positivo) y agua libre de nucleasas (negativo).

Aspectos éticos. El presente estudio se realizó conforme a los principios emanados de la ley nacional 25.326/2000 (Protección de los datos personales), la ley de CABA 3.301/2009 (Protección de Derechos de Sujetos en Investigaciones en Salud) y la Declaración de Helsinki (1964) de la Asociación Médica Mundial y actualizaciones posteriores. También estuvo conforme a los "Principios rectores internacionales aplicables a las investigaciones biomédicas con animales" del Consejo de Organizaciones
Internacionales de las Ciencias Médicas aprobado por el Comité Consultivo de Investigaciones Médicas de la Organización Mundial de la Salud (1985), las "Normas Internacionales para la investigación biomédica con animales" de la Organización Panamericana de la Salud (1990) y el Título 7: "Bienestar de los animales" del Código Sanitario para los Animales Terrestres de la Organización Mundial de Sanidad Animal (2011). El proyecto contó con la aprobación del Comité de Docencia e Investigación del Instituto de Zoonosis Luis Pasteur.

\section{RESULTADOS}

Se procesaron 422 ejemplares de las especies $A$. aureolatum, A. brasiliense, A. ovale, A. sculptum, A. tigrinum, $A$. tonelliae y $A$. triste. En la Tabla 1 se presenta el detalle por especie, estadio, hospedador (o vegetación en el caso de aquellas colectas en su fase de vida libre) y localidad de colecta.

Todas las muestras analizadas resultaron negativas a la PCR para el fragmento del gen fla del género Borrelia.

Tabla 1. Especie, localidad de procedencia, fuente de colecta (hospedadores/vegetación) y número de especímenes analizados de las garrapatas estudiadas

\begin{tabular}{|c|c|c|c|c|c|c|}
\hline Especie & Provincia & $\begin{array}{c}\text { Localidad } \\
\text { (Coordenadas geográficas) }\end{array}$ & $\begin{array}{l}\text { Hospedador/ } \\
\text { vegetación }\end{array}$ & Estadio & $\mathbf{n}$ & Total \\
\hline \multirow{7}{*}{ Amblyomma aureolatum } & \multirow{7}{*}{ Entre Ríos } & Colón $\left(32^{\circ} 13^{\prime} \mathrm{S}, 58^{\circ} 08^{\prime} \mathrm{O}\right)$ & Perro & Adulto & 4 & \multirow{7}{*}{37} \\
\hline & & Feliciano $\left(30^{\circ} 23^{\prime} \mathrm{S}, 58^{\circ} 45^{\prime} \mathrm{O}\right)$ & Perro & Adulto & 2 & \\
\hline & & \multirow{2}{*}{ La Paz $\left(30^{\circ} 45^{\prime} S, 5^{\circ} 39^{\prime} \mathrm{O}\right)$} & Perro & Adulto & 8 & \\
\hline & & & Gato & Adulto & 1 & \\
\hline & & \multirow{3}{*}{ Paraná $\left(31^{\circ} 43^{\prime} \mathrm{S}, 60^{\circ} 31^{\prime} \mathrm{O}\right)$} & Perro & Adulto & 19 & \\
\hline & & & Gato & Adulto & 2 & \\
\hline & & & Humano & Adulto & 1 & \\
\hline \multirow{4}{*}{ Amblyomma brasiliense } & \multirow{4}{*}{ Misiones } & \multirow{2}{*}{$\begin{array}{l}\text { Parque Nacional Iguazú } \\
\left(25^{\circ} 41^{\prime} \mathrm{S}, 54^{\circ} 27^{\prime} \mathrm{O}\right)\end{array}$} & Vegetación & Adulto & 47 & \multirow{4}{*}{92} \\
\hline & & & Vegetación & Ninfa & 35 & \\
\hline & & \multirow{2}{*}{$\begin{array}{l}\text { Parque Provincial Puerto Península } \\
\left(25^{\circ} 43^{\prime} \mathrm{S}, 54^{\circ} 33^{\prime} \mathrm{O}\right)\end{array}$} & Vegetación & Adulto & 5 & \\
\hline & & & Vegetación & Ninfa & 5 & \\
\hline \multirow{3}{*}{ Amblyomma ovale } & \multirow{3}{*}{ Formosa } & \multirow{3}{*}{ El Bagual $\left(25^{\circ} 59^{\prime} S, 58^{\circ} 24^{\prime} \mathrm{O}\right)$} & Vegetación & Ninfa & 2 & \multirow{3}{*}{8} \\
\hline & & & \multirow{2}{*}{$\begin{array}{l}\text { Taraba major } \\
\text { (Chororó) }\end{array}$} & Ninfa & 4 & \\
\hline & & & & Larva & 2 & \\
\hline Amblyomma sculptum & Salta & $\begin{array}{l}\text { Parque Nacional El Rey } \\
\left(24^{\circ} 15^{\prime} \mathrm{S}, 64^{\circ} 40^{\prime} \mathrm{O}\right)\end{array}$ & Vegetación & Adulto & 80 & 80 \\
\hline \multirow{5}{*}{ Amblyomma tigrinum } & \multirow{3}{*}{ Córdoba } & \multirow{2}{*}{ Nono $\left(31^{\circ} 46^{\prime} \mathrm{S}, 64^{\circ} 59^{\prime} \mathrm{O}\right)$} & Perro & Adulto & 4 & \multirow{5}{*}{99} \\
\hline & & & Vegetación & Adulto & 11 & \\
\hline & & Salsipuedes $\left(31^{\circ} 09^{\prime} \mathrm{S}, 64^{\circ} 19^{\prime} \mathrm{O}\right)$ & Vegetación & Adulto & 42 & \\
\hline & \multirow{2}{*}{ San Luis } & Cortaderas $\left(32^{\circ} 30^{\prime} \mathrm{S}, 65^{\circ} 00^{\prime} \mathrm{O}\right)$ & Gato & Adulto & 19 & \\
\hline & & Merlo $\left(32^{\circ} 20^{\prime} \mathrm{S}, 65^{\circ} 00^{\prime} \mathrm{O}\right)$ & Perro & Adulto & 23 & \\
\hline Amblyomma tonelliae & Salta & El Tunal $\left(25^{\circ} 14^{\prime} \mathrm{S}, 64^{\circ} 25^{\prime} \mathrm{O}\right)$ & Vegetación & Adulto & 52 & 52 \\
\hline \multirow[t]{2}{*}{ Amblyomma triste } & \multirow[t]{2}{*}{ Buenos Aires } & $\begin{array}{l}\text { INTA E.E.A. Delta del Paraná } \\
\left(34^{\circ} 11^{\prime} \mathrm{S}, 58^{\circ} 50^{\circ} \mathrm{O}\right)\end{array}$ & Vegetación & Adulto & 35 & \multirow[t]{2}{*}{54} \\
\hline & & General Lavalle $\left(36^{\circ} 22^{\prime} \mathrm{S}, 56^{\circ} 21^{\prime} \mathrm{O}\right)$ & Vegetación & Adulto & 19 & \\
\hline Total & & & & & & 422 \\
\hline
\end{tabular}




\section{DISCUSIÓN}

Este es el primer estudio que evalúa la presencia de Borrelia spp. en garrapatas $A$. sculptum, $A$. tigrinum y $A$. tonelliae y el primero en Argentina para las especies estudiadas (con excepción de A. triste y $A$. aureolatum).

Hasta el presente en Argentina el estudio de Borrelia en el género Amblyomma se limitó a un único trabajo en el que Cicuttin et al (3) analizaron 432 garrapatas $A$. aureolatum y 65 A. triste colectadas en la Reserva Ecológica Costanera Sur de la Ciudad Autónoma de Buenos Aires, encontrando $1.7 \%$ de $A$. aureolatum positivas a una Borrelia sp. filogenéticamente relacionada al grupo REP. En dicho estudio, sólo ninfas de $A$. aureolatum resultaron positivas ( $9 / 52-17.3 \%$ - en ninfas colectadas sobre vegetación y 5/11 -45.4\%- en las colectadas sobre aves), destacándose el posible rol de las aves como hospedadores amplificadores de la Borrelia sp. REP hallada (3). Del mismo modo, los hallazgos de Borrelia sp. REP en Brasil se realizaron en ninfas de $A$. longirostre colectadas sobre aves (5), mientras que en Guayana Francesa se detectó en larvas de $A$. longirostre y $A$. geayi parasitando aves (8) y en México se detectó en ninfas y adultos de $A$. dissimile colectadas de sapos (9). Por otro lado, el reporte de Borrelia sp. del grupo REP en EEUU se obtuvo a partir de adultos de $A$. maculatum de vida libre colectados en vegetación (4). Las genoespecies de Borrelia del grupo REP halladas en EEUU, Brasil y Guayana Francesa están estrechamente relacionadas entre sí (posiblemente la misma especie) desde una perspectiva filogenética, pero no con la Borrelia sp. del grupo REP hallada en $A$. aureolatum de Argentina (8). La Borrelia sp. recientemente hallada en $A$. brasiliense de Brasil se relaciona con secuencias de Borrelia spp. reportadas en Etiopía y Costa de Marfil, formando un grupo separado de REP y GFR (10). En ese estudio se detectó Borrelia sp. en 1 grupo de 6 ninfas colectadas de vegetación (de 266 estudiadas) (10); en comparación en el presente estudio se analizaron sólo 40 ninfas de $A$. brasiliense.
Respecto a la asociación entre el GBB y el género Amblyomma, una amplia revisión realizada por Stromdahl et al (17) demuestra que los escasos hallazgos positivos $(0,1 \%$ en casi 30.000 ejemplares estudiados) en $A$. americanum de EEUU corresponden a estudios en garrapatas colectadas sobre hospedadores (el ADN detectado está posiblemente relacionado a la ingesta de sangre infectada desde el hospedador) o a sospecha de contaminación en el laboratorio. Por otra parte, en una revisión sobre estudios experimentales para determinar la competencia vectorial de $A$. americanum en la transmisión de $B$. burgdorferi s.l., se concluye que esta especie contribuye mínimamente o de ninguna forma al ciclo de transmisión de la borreliosis de Lyme en dicho país (18). La presencia de péptidos antimicrobianos (defensinas) con actividad lítica frente a $B$. burgdorferi s.I. ha sido considerado como un factor importante (18).

Por último, diversos estudios en $A$. americanum de EEUU reportan 1-3\% de infección por Borrelia lonestari, especie perteneciente al GFR (7).

En el presente estudio no se detectó ADN de bacterias del género Borrelia en diferentes especies de garrapatas de importancia para la salud pública pertenecientes al género Amblyomma. Considerando los escasos antecedentes previos, futuros estudios en esta temáticas son necesarios, especialmente para el caso de especies de garrapatas que parasitan frecuentemente a aves, reptiles y anfibios, dado que estos hospedadores podrían actuar como potenciales reservorios y amplificadores en el ciclo enzoótico de las borrelias asociadas a Amblyomma en la región.

\section{Conflictos de intereses}

Los autores declaran no tener conflicto de intereses.

\section{Agradecimientos}

El presente trabajo ha sido parcialmente financiado por el Subsidio para Investigación Clínica y Epidemiológica 2018 de la Fundación Alberto J. Roemmers. 


\section{REFERENCIAS}

1. Nava S, Venzal JM, González Acuña D, Martins TF, Guglielmone AA. Ticks of the Southern Cone of America: Diagnosis, Distribution and Hosts with Taxonomy, Ecology and Sanitary Importance. London: Academic Press; 2017.

2. Margos G, Gofton A, Wibberg D, Dangel A, Marosevic D, Loh SM, et al. The genus Borrelia reloaded. PLoS One. 2018; 13(12):1-14. https://doi.org/10.1371/ journal.pone.0208432

3. Cicuttin GL, De Salvo MN, Venzal JM, Nava S. Borrelia spp. in ticks and birds from a protected urban area in Buenos Aires city, Argentina. Ticks Tick Borne Dis. 2019; 10(6): 101282. https://doi.org/10.1016/j. ttbdis.2019.101282

4. Lee JK, Smith WC, McIntosh C, Ferraric FG, Moore Hendersond B, Varela Stokes A. Detection of a Borrelia species in questing Gulf Coast ticks, Amblyomma maculatum. Ticks Tick Borne Dis. 2014; 5(4):449-452. https://doi.org/10.1016/j. ttbdis.2014.02.004

5. Pacheco A, Cordeiro MD, Cepeda MB, Luz HR, Cardozo SV, Berto BP, et al. Hemoparasites in ticks of wild birds of Serra dos Órgãos National Park, state of Rio de Janeiro, Brazil. Brazilian J Vet Parasitol. 2019; 28(2). https://doi.org/10.1590/ s1984-29612019017

6. Barbour AG, Maupin GO, Teltow GJ, Carter CJ, Piesman J. Identification of an uncultivable Borrelia species in the hard tick Amblyomma americanum: possible agent of a Lyme disease-like illness. J Infect Dis. $1996 ; 173(2): 403-409$. https://doi. org/10.1093/infdis/173.2.403

7. James AM, Liveris D, Wormser GP, Schwartz I, Montecalvo M a, Johnson BJ. Borrelia lonestari infection after a bite by an Amblyomma americanum tick. J Infect Dis. 2001; 183 (12):1810-1814. https://doi. org/10.1093/infdis/173.2.403
8. Binetruy $F$, Garnier S, Boulanger N, Talagrand-reboul É, Loire E, Faivre B, et al. A novel Borrelia species, intermediate between Lyme disease and relapsing fever groups, in neotropical passerine-associated ticks. Sci Rep. 2020; 10:10596. https://doi. org/10.1038/s41598-020-66828-7

9. Colunga-Salas $P$, Sánchez-Montes $S$, Ochoa-Ochoa LM, Grostieta E, Becker I. Molecular detection of the reptile-associated Borrelia group in Amblyomma dissimile, Mexico. Medical and Veterinary Entomology. 2020; 52:1-5. https://doi.org/ 10.1111/ mve. 12478

10. dos Santos $C A$, Suzin $A$, Vogliotti $A$, Nunes $\mathrm{PH}$, Barbieri ARM, Labruna MB, et al. Molecular Detection of a Borrelia sp. in nymphs of Amblyomma brasiliense ticks (Acari: Ixodidae) from Iguaçu National Park, Brazil, genetically related to Borrelia from Ethiopia and Côte D'Ivoire. Ticks Tick Borne Dis. 2020; 11(6):101519. https:// doi.org/10.1016/j.ttbdis.2020.101519

11. Cicuttin G, Nava S. Molecular identification of Rickettsia parkeri infecting Amblyomma triste ticks in an area of Argentina where cases of rickettsiosis were diagnosed. Mem Inst Oswaldo Cruz. 2013; 108(1):123125. http://dx.doi.org/10.1590/S0074$\underline{02762013000100022}$

12. Cicuttin GL, De Salvo MN, Nava S. Two novel Ehrlichia strains detected in Amblyomma tigrinum ticks associated to dogs in periurban areas of Argentina. Comp Immunol Microbiol Infect Dis. 2017; 53:40-44. https:// doi.org/10.1016/j.cimid.2017.07.001

13. Cicuttin $G L$, Salvo $M N$ De, Pérez PD, Silva D, Félix L, Venzal JM, et al. A novel Ehrlichia strain (Rickettsiales: Anaplasmataceae) detected in Amblyomma triste (Acari: Ixodidae), a tick species of public health importance in the Southern Cone of America. Pathog Glob Health. 2020; 114(6):318-322. https://doi.org/10.1080/20477724.2020.1 $\underline{795579}$ 
14. Lamattina D, Tarragona EL, Nava S. Molecular detection of the human pathogen Rickettsia parkeri strain Atlantic rainforest in Amblyomma ovale ticks in Argentina. Ticks Tick Borne Dis. 2018; 9(5):12611263. https://doi.org/10.1016/j. ttbdis.2018.05.007

15. Mastropaolo M, Tarragona EL, Silaghi C, Pfister K, Thiel C, Nava S. High prevalence of "Candidatus Rickettsia amblyommii" in Amblyomma ticks from a Spotted Fever Endemic Region in North Argentina. Comp Immunol Microbiol Infect Dis. 2016; 46:73-76. http://dx.doi.org/10.1016/j. cimid.2016.05.001

16. Tarragona EL, Cicuttin GL, Mangold AJ, Mastropaolo M, Salvo MN De, Nava S. Rickettsia infection in Amblyomma tonelliae, a tick species from the Amblyomma cajennense complex. Ticks Tick Borne Dis. 2014; 6(2):173-177. http://doi. org/10.1016/j.ttbdis.2014.11.010
17. Stromdahl EY, Nadolny RM, Hickling GJ, Hamer SA, Ogden NH, Casal C, et al. Amblyomma americanum (Acari: Ixodidae) ticks are not vectors of the Lyme Disease agent, Borrelia burgdorferi (Spirocheatales: Spirochaetaceae): A review of the evidence. J Med Entomol. 2018; 55(3):501-514. http://doi.org/10.1093/jme/tjx250

18. Eisen L. Vector competence studies with hard ticks and Borrelia burgdorferi sensu lato spirochetes: A review. Ticks Tick Borne Dis. $2020 ; 11(3): 101359$. https://doi. org/10.1016/j.ttbdis.2019.101359 